\title{
Molecular Dynamics Simulation of the Effects of Methane Hydrate Phase Transition on Mechanical Properties of Deep-Sea Methane Hydrate-Bearing Soil
}

\author{
Yanmei Zhang, ${ }^{1}$ Jian Zhang $\mathbb{D}^{1,2}$ Guoxun Li, ${ }^{1}$ Changda Sun, ${ }^{1}$ Yalin Luan, ${ }^{1}$ \\ and Jianlin Liu $\mathbb{D D}^{1}$ \\ ${ }^{1}$ College of Pipeline and Civil Engineering, China University of Petroleum (Huadong), Qingdao 266580, China \\ ${ }^{2}$ China Communications Construction Company, Ltd., Beijing 100088, China \\ Correspondence should be addressed to Jian Zhang; 416126038@qq.com
}

Received 12 September 2021; Revised 8 November 2021; Accepted 18 November 2021; Published 2 December 2021

Academic Editor: Angelo Aloisio

Copyright (C) 2021 Yanmei Zhang et al. This is an open access article distributed under the Creative Commons Attribution License, which permits unrestricted use, distribution, and reproduction in any medium, provided the original work is properly cited.

\begin{abstract}
In this paper, the methane hydrate phase transition process in deep-sea methane hydrate-bearing soil under heating and compression was simulated by the molecular dynamics method. The evolution of deep-sea methane hydrate-bearing soil's microstructure, system energy, intermolecular interaction energy, and radial distribution function during heating and compression was investigated. The micromechanism of the influence of the methane hydrate phase transition on the mechanical properties of deep-sea methane hydrate-bearing soil was analyzed. The results demonstrated that the methane hydrate dissociation starts from both sides to the middle and the void spaces between the soil particles had nearly no change during the heating process. For the compression process, the methane hydrate on both sides and the middle dissociated at the same time, and the void spaces became smaller. The methane hydrate phase transition on the effects of mechanical properties of the deep-sea methane hydrate-bearing soil is mainly caused by three aspects. (1) the dissociation of methane hydrate incurs the decrease of methane hydrate saturation. The free water and methane molecules generated cannot migrate in time and thus lead to the increase of excess pore water press and excess pore gas press. (2) The dissipated energy causes the decrease of the effective stress between the soil particles. (3) Due to the methane hydrate decomposition, the free water molecules increase, which reduces the friction of soil particles.
\end{abstract}

\section{Introduction}

Methane hydrates, ice-like substances, are formed in the deep-sea soil with high-pressure and low-temperature conditions especially under the seabed on the continental slopes. For simplicity, the sediments containing natural methane hydrate in the deep-sea soil are defined as deep-sea methane hydrate-bearing soil (MHBS) [1]. It is a kind of multiphase and multicomponent complex porous medium. Its mechanical behaviors have strong nonlinearity and complicated structural properties and have a significant correlation to temperature and pressure [2]. The exploitation of methane hydrates, which is related to methane hydrates dissociation, can easily lead to the deterioration of mechanical properties of MHBS [3]. There are usually four suggested methods to produce methane by methane hydrates dissociation: depressurizing, thermal stimulation, chemical injection, and gas swapping [4]. While depressurization is used for gas production, MHBS is consolidated due to the increasing effective stress, which could trigger marine slope failure and increase the risk of tsunamis [5]. Considering the gas diffusion and fluid expansion, high pore fluid pressure generation is anticipated because of the thermal stimulation [6]. As a result, a number of massive slides have been caused by the methane hydrate decomposition [7]. Currently, these problems have attracted increasing interests of the researchers all over the world. Kataoka et al. [8], Santamarina [9], Puppel et al. [10], and 
Francisca et al. [11] studied the effects of methane hydrates on the physical and mechanical properties of MHBS by site sampling and experiments. The results indicated that the mechanical properties of MHBS deteriorated after the decomposition of methane hydrates. Kajiyama et al. [12] examined the mechanical properties of MHBS with methane hydrate-bearing sands generated with rounded glass beads and natural sands in the lab. The effects of the particle characteristics on the mechanical response of MHBS were studied from a grain-scale viewpoint. Based on several empirical relationships of granular materials, Wu et al. [13] proposed a simple constitutive model to describe the stressstrain relationship of MBHS, which could model the mechanical properties of MBHS. Holtzman et al. [14] built a constitutive model of MHBS to evaluate the effects of methane hydrate dissociation by variational approaches. Several possible dissociation scenarios were calculated by this model. It was shown that the solid support of the skeleton could be lost due to the methane hydrate dissociation, which could cause seafloor landslide and subsidence. Considering the critical role of the stability of MHBS to the safe exploitation of methane gas, Wu et al. [15] improved the previous MHBS constitutive models by introducing hydrate saturation. A new three-dimensional critical state surface was identified, and it could be used to determine the state parameters of MHBS. Rutqvist and Moridis [16], Xu and Germanovich [17], and Jiang and Gong [18] simulated the methane hydrate decomposition process by establishing a hydrate cementation model using discrete element method. The results showed that the hydrate dissociation has significant impacts on the mechanical properties of MHBS. Li [19] observed the formation of methane hydrate in sands on pore-scale by a nano-focus X-ray computed tomography (X$\mathrm{CT}$ ). The gas/water/hydrate distributions and the pore structure characteristics were investigated. Kneafsey et al. [20] performed a series of tests to investigate the heat transfer and the hydrate dissociation kinetics on a partially saturated sand. Throughout the processes, both the temperature and the pressure played a vital role.

As mentioned above, the deterioration of mechanical properties of MHBS is mainly caused by the dissociation of the methane hydrate. Therefore, a deep understanding of methane hydrate phase transition on the effects of mechanical properties of MHBS especially from a microperspective is beneficial to study and explain their macromechanical properties. However, monitoring and controlling the methane hydrate formation and dissociation processes through experimental works are very difficult and it is nearly impossible to reach equal conditions as the deep marine environments [21]. To overcome the current limitations of sampling and experimentation, molecular dynamic (MD) simulations have been utilized to investigate the growth, nucleation, structure, dissociation, and thermodynamic and mechanical characteristics of methane hydrate. Ji et al. explored the formation mechanisms and decomposition strategies of methane hydrates in the heterogeneous sediment environments with the MD model [22]. Li et al. studied the effects of different thermodynamic parameters, temperatures, pressure, and gas concentrations on methane hydrate dissociation with the MD method [23]. Wang et al. investigated the microstructure changes and mechanical properties of methane hydrate under the condition of compression and tension by using MD simulation [24]. Accurately understanding the microscopic deterioration mechanism could help us to exploit and utilize MHBS in the right way. Nonetheless, most of the studies were focused on methane hydrate dissociation and dissociation processes. The effects of methane hydrate phase transition on the mechanical properties of MHBS need to be more explored.

In this paper, the microstructure evolutions of MHBS under heating and compression processes were simulated with the MD model. The details of phase transition and water and methane molecules movements during the methane hydrate phase transition processes were observed. The microscopic mechanism of the deterioration of mechanical properties of MHBS was analyzed.

\section{Model and Computational Method}

2.1. Simulation Model. The structure I hydrate unit, which consists of two $5^{12}$ small cages and six $5^{12} 6^{2}$ large cages, was selected to form a methane hydrate model in this study [25]. Its cubic unit contained 8 methane molecules and 46 water molecules arranged in the cubic box of $12 \times 12 \times 12 \AA^{3}$. The simple point-charge (SPC) model was selected for water. A silica surface was abstracted to represent the real geologic conditions [26]. The quartz slab was generated with a sequence of $\alpha$-quartz formed by an orthorhombic unit cell, and then, a fully coordinated surface (Si-O-Si bridged surface) was created by a series of quartz slabs. The $\alpha$-quartz was characterized by a hexagonal structure with a space group $\mathrm{P}_{3}{ }_{2} 21$. The lattice parameters were $A=B=4.904 \AA$ and $C=5.4218 \AA$ [27]. In total, $1 \times 1 \times 4$ supercells of structure I hydrate and two $3 \times 3 \times 10$ of $\alpha$-quartz layers were combined to investigate the effects of the microscopic mechanism of the methane hydrate dissociation and phase transition process under heating and compression conditions. Because MHBS was water saturated, the surfaces of quartz layers were hydroxylated [28]. To build the hydrophilic silica surface, the bulk crystal of $\alpha$-quartz along the [llll $\left.\begin{array}{lll}0 & 1\end{array}\right]$ direction was cleaved. After that, on the fresh silica surface, $\mathrm{H}$ atoms were manually added, and the $\mathrm{H}$ atoms were linked with the $\mathrm{O}$ atoms [26]. Finally, the simulation model of deep-sea methane hydrate-bearing soil was formed as shown in Figure 1. The top and bottom layers were $\alpha$-quartz, and the middle layer was the hydrate layer. The cell length was16, 45 , and $70 \AA$ in the $x, y$, and $z$ directions, respectively.

The MD simulations were performed using LAMMPS, which is open-source software for parallel simulations. The Lennard-Jones potential was adopted as the force field, where

$$
\mu\left(r_{i j}\right)=\frac{C q_{i} q_{j}}{\varepsilon r_{i j}}+4 \varepsilon\left[\left(\frac{\sigma}{r_{i j}}\right)^{12}+\left(\frac{\sigma}{r_{i j}}\right)\right]^{6},
$$

where $\mu$ is the potential energy; $C$ is an energy-conversion constant, $q$ is the partial charge, and $\varepsilon$ is the dielectric constant. $\mathbf{r}_{\mathbf{i j}}$ is the distance between two interplay particles $i$ 


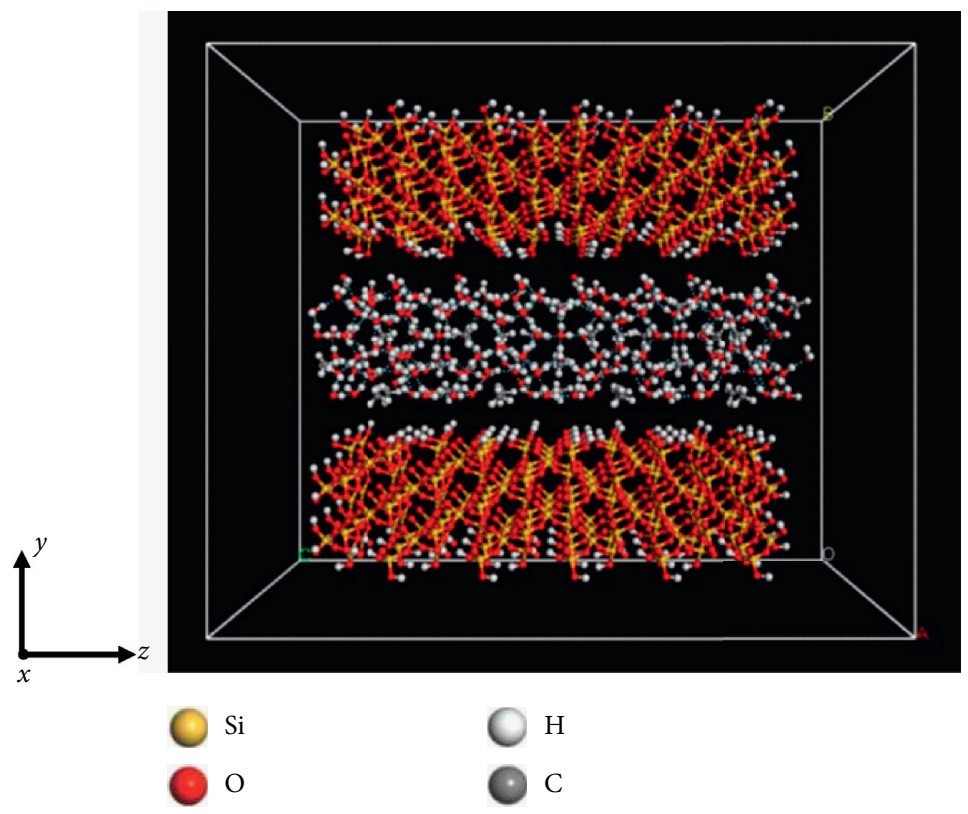

Figure 1: Simulation model of deep-sea methane hydrate-bearing soil.

and $j$, and $\sigma$ is the distance at which the particle-particle energy is zero [24]. The force field parameters are given in Table $1[25,27,29]$.

2.2. Computational Parameters. The simulation systems were established based on energy minimization. Periodic boundary conditions were used in the three directions for all simulations. The cutoff radius for short-range interaction was $9.5 \AA$, and the long-range Coulomb interactions were calculated using the PPPM algorithm. Before the heating and compression processes, the quartz layers were fixed. After that, the NPT ensemble was used to relax for $50 \mathrm{fs}$ at $273 \mathrm{~K}$ with the time Step $0.001 \mathrm{fs}$. The pressure in the $x$-direction and $z$-direction was controlled at $3 \mathrm{MPa}$ while it was free in the $y$-direction, which was used to simulate the environment 300 meters under sea level. The system reached the equilibrium state, loosened the fixed quartz layers, and made the whole system achieve equilibrium. As shown in Figure 2, the energy of the whole system reached equilibrium at $50 \mathrm{fs}$. Afterward, the heating or compression processes were conducted. The total simulation time was $600 \mathrm{fs}$ with time Step $0.001 \mathrm{fs}$. During the heating process, the temperature increased from $273 \mathrm{~K}$ to $300 \mathrm{~K}$ in $600 \mathrm{fs}$. For the compression process, the model was tested with a strain rate of $0.001 / \mathrm{fs}$ under a constant temperature of $273 \mathrm{~K}$.

\section{Results and Discussion}

3.1. Effects of Temperature. Figure 3 shows the microscopic structure evolution of MHBS with the temperature rising. Figure 3(a) was the initial stage when the temperature was $273 \mathrm{~K}$ and the cage structures of methane hydrate were complete. As the temperature increased to $276 \mathrm{~K}$, the methane hydrate began to dissociate (Figure $3(\mathrm{~b})$ ). The cage structures, which were located on the upper and lower sides of methane hydrate, began to dissociate at the same time, and then, a passage was formed by the damaged cage structures (Figures 3(b) and 3(c)). The methane molecules moved outside as bubbles along the passage. Due to the constraint of the quartz layers on both sides, the methane molecules moved only through the $z$-direction. After that, the decomposition of the methane hydrate moved from both sides to the middle part as the temperature rose. As shown in Figure $3(\mathrm{~d})$, the hydrate cage structure in the middle remained relatively intact when the temperature reached $300 \mathrm{~K}$. In addition, it could be observed in Figure 3 that the void spaces between the soil particles did not change, and the molecular structure of quartz layers changed slightly. This was because even though the temperature rose and the molecular motion intensified, the molecular structure of quartz layers remained relatively intact due to the quartz layers' strong molecular interactions.

In conclusion, the microstructural evolution and the migration law of methane molecules under rising temperature can be described as follows: the methane hydrate inside MHBS firstly dissociates from both sides because the heat spreads from outside to inside with rising temperature. The methane molecules escape while the cage structures are destroyed. As the temperature continues to increase, the cage structure in the middle part begins to break and then a passage is formed together with the notch generated on both sides. After that, the methane molecules inside the middle part of MHBS escape through the passage. However, there are still a small number of methane molecules left inside the MHBS when the heating process is finished, which is named soil gas. Since the methane hydrate decomposes from both sides to the middle part and the voids of the soil are relatively stable, the rising temperature is beneficial for methane hydrate exploitation. 
TABLE 1: Lennard-Jones potential parameters [25, 27, 29].

\begin{tabular}{|c|c|c|c|c|}
\hline Molecule & Atom & $\varepsilon(\mathrm{kJ} / \mathrm{mol})$ & $\sigma(\AA)$ & $q(\mathrm{e})$ \\
\hline \multirow{2}{*}{$\mathrm{H}_{2} \mathrm{O}$} & $\mathrm{O}$ & 0.1555 & 3.488 & -0.82 \\
\hline & $\mathrm{H}$ & 0 & 0 & 0.41 \\
\hline \multirow{2}{*}{$\mathrm{CH}_{4}$} & $\mathrm{C}$ & 0.066 & 3.5 & -0.4 \\
\hline & $\mathrm{H}$ & 0.03 & 2.5 & 0.41 \\
\hline \multirow{4}{*}{$\alpha$-quartz } & $\mathrm{Si}$ & 0.5335 & 3.795 & 2.4 \\
\hline & $\mathrm{O}\left(\mathrm{SiO}_{2}\right)$ & 0.6487 & 3.154 & -0.82 \\
\hline & $\mathrm{H}$ (at $\alpha$-quartz boundary) & 0 & 0 & 0.41 \\
\hline & $\mathrm{O}$ (at $\alpha$-quartz boundary) & 0.1555 & 3.488 & -0.82 \\
\hline
\end{tabular}

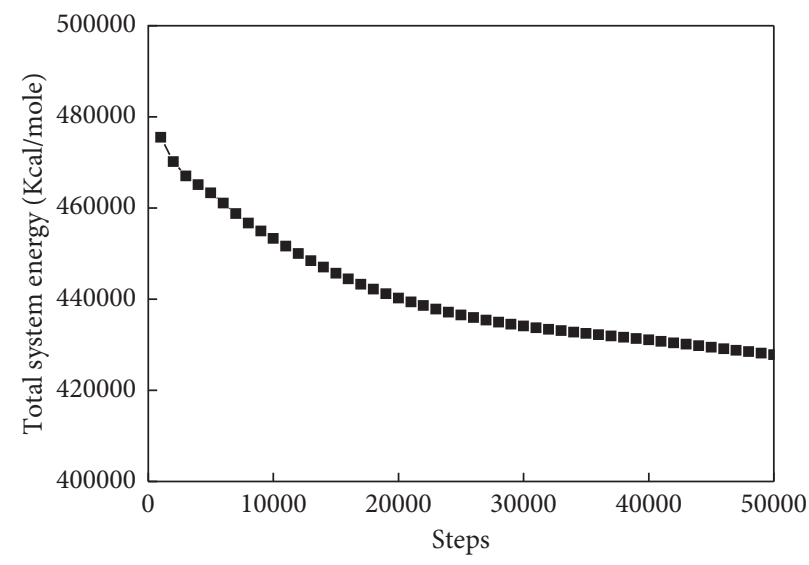

FIGURE 2: Equilibrium of energy in the initial system.
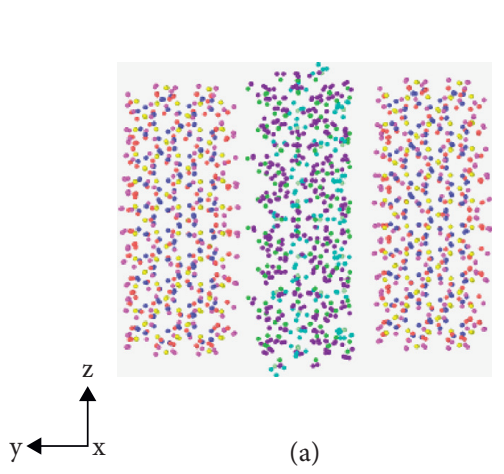

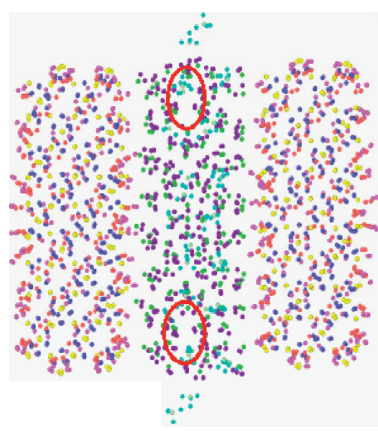

(b)

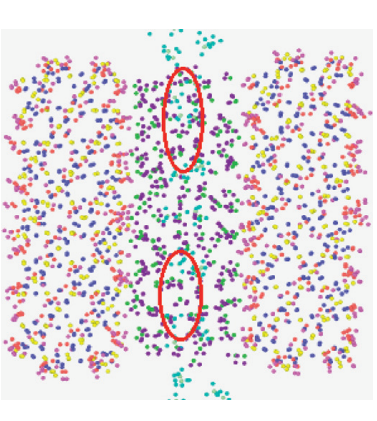

(c)

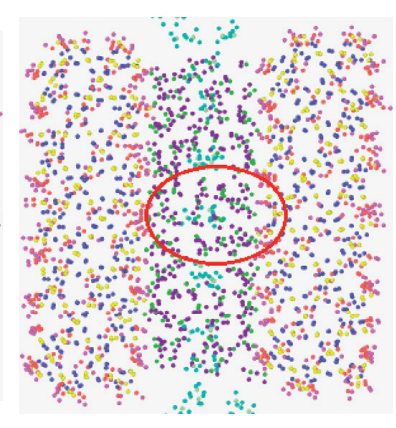

(d)

Figure 3: Microstructure changes of MHBS during the heating process. (a) Initial stage. (b) Methane hydrate dissociation. (c) Methane hydrate migration. (d) The stage at $300 \mathrm{~K}$.

Figure 4 shows the stress evolution of MHBS with increasing temperature. The stress in the $y$-direction kept decreasing as the temperature increased. At the initial stage, the stress decreased at a higher rate, which meant that the methane hydrate was sensitive to temperature. With the escape of methane molecules after hydrate dissociation, the methane hydrate saturation decreased, which led to a decrease in soil strength. Meanwhile, due to the limitation of adjacent soil particles $s$ and the tendency for volume expansion associated with methane hydrate dissociation, methane and water liberated were aggregated and caused excess pore water pressure and excess pore gas pressure in the system. Excess pore pressure within MHBS can result in a mechanical weakening of the MHBS $[17,30]$. After that, the stress kept decreasing at a relatively stable rate. Since the methane hydrate was beneficial to the strength of MHBS, it was reasonable that the strength of MHBS decreased after the methane hydrates were dissociated. The stress nearly disappeared after the massive decomposition of hydrates when the temperature reached $281 \mathrm{~K}$.

Figure 5 shows the energy evolution during the heating process, where the negative sign represents the interatomic attraction between atoms. In Figure 5(a), the energy of the total system gradually decreased during the heating process. It was obvious that the energy decreasing rate at the initial stage of methane hydrate decomposition $(<285 \mathrm{~K})$ was faster due to the energy lost with the escaping methane molecules during the heating process. Considering the effects of 


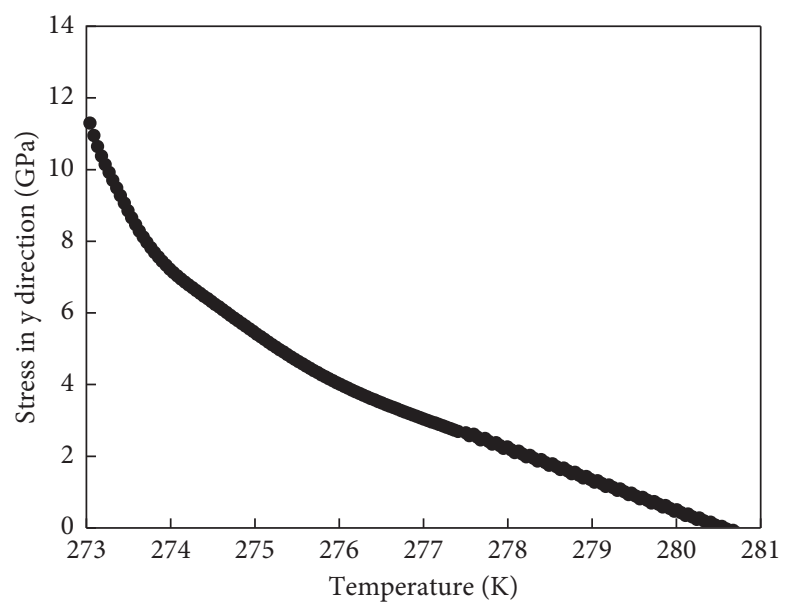

FIgURE 4: Stress evolution of MHBS in the $y$-direction during the heating process.

modeling, the microscopical model represents only "one point." The macroscopical materials are formed by a series of "one point." So, the energy dissipated from "one point" could work on the neighborhood point or be released outside. While the energy released from "one point" and "one point" is applied to the MHBS, the effective stress between the soil particles decreases.

Figure 5(b) shows the intermolecular interaction energy variation between silicon atoms and water molecules, and methane molecules and methane molecules during the heating process. The interaction energy between silicon atoms and water molecules increased with increasing temperature, which indicated that the free water molecules generated during the heating process kept interacting with quartz layers. The interaction increased with the rise in temperature. This was consistent with the phenomenon in macroscale, where the free water increased and affected the soil particle's friction. The mechanical properties of MHBS decreased consequently. In addition, Figure 5(b) shows that the interaction energy between methane molecules and methane molecules decreased gradually with increasing temperature. The reduction was small and stable, which was consistent with the escape of methane molecules through the passage formed in MHBS.

Therefore, during the heating process, the effects of changes in methane hydrate on the mechanical properties of MHBS mainly contain three aspects. Firstly, the water and methane molecules generated by the decomposition of methane hydrate cannot escape due to the constrain of the soil particles, which results in excess pore water pressure and excess pore gas pressure, and thus, the MHBS strength decreases. Secondly, the dissipated energy cannot be released outside and accumulated in the soil. While it works on the soil particles, the effective stress between the soil particles decreases. Thirdly, the free water molecules generated from the hydrate dissociation were equal to the addition of lubricants between soil particles and thus decreased the friction of the soil particles.

Figure 6(a) shows the radial distribution function (RDF) of silica atoms in quartz and oxygen atoms in the water molecules of methane hydrate. Due to the high thickness of quartz layers, water molecules were less likely to appear within the statistical radius. However, according to RDF, the water molecules surrounding the quartz layers kept increasing. It could be explained by the fact that the free water molecules generated from methane hydrate decomposition moved to the quartz layer. The quartz layers constrained water molecules' movement and thus they interacted with each other. This was consistent with the increasing interaction energy between silica atoms and water molecules (Figure 5(b). In addition, two peak values were observed except at temperature $280 \mathrm{~K}$. The disappeared peak value was caused by the fact that the quartz layers' hydroxy surfaces were dissociated while the temperature increased to $280 \mathrm{~K}$, and the water and methane molecules still did not escape at this time. This was also why the RDF had the highest value under $280 \mathrm{~K}$. Figure 6 (b) shows the carbon atom distribution to reflect the distribution of methane molecules. There were multiple peaks, which indicated that methane molecules were tightly packed. The highest value came out at $280 \mathrm{~K}$ because the water and methane molecules still did not migrate at this time. It was also consistent with the fact that the methane molecules migrated and escaped only through the passage in the $Z$-direction.

3.2. Effects of Compression. Figure 7 shows the microscopic structure evolution of MHBS during the compression process. Figure 7(a) was the initial stage of the compression process. As the compression process started (Figure 7(b)), the methane hydrate began to dissociate, and a passage was formed as the hydrate cage structure was destroyed simultaneously. The methane molecules at both sides began to escape. As the compression process continued (Figure 7(c)), the dissociated methane molecules from the middle of the hydrate began to move outside from the passage and the saturation of MHBS decreased. While the compression processes were completed (Figure $7(\mathrm{~d})$ ), all the hydrate cage structures were destroyed. The free water molecules and methane molecules generated coexisted inside MHBS. Obviously, the void spaces between the soil particles decreased, and the quartz layers were kept relatively stable 


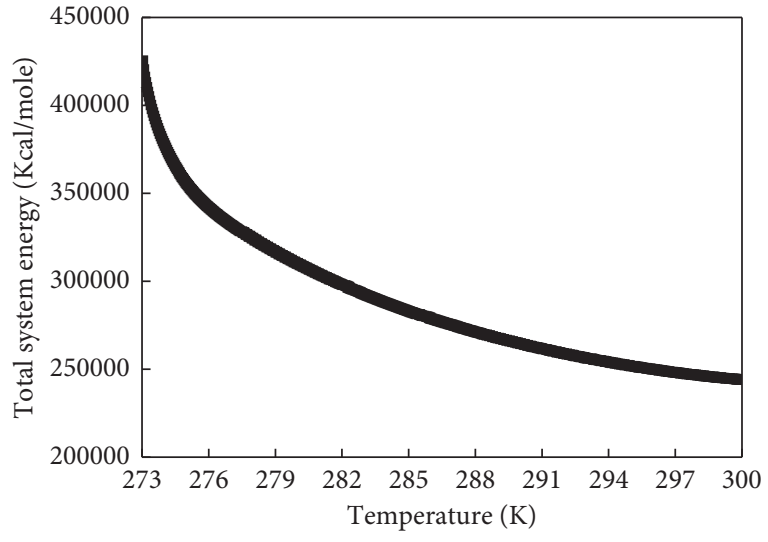

(a)

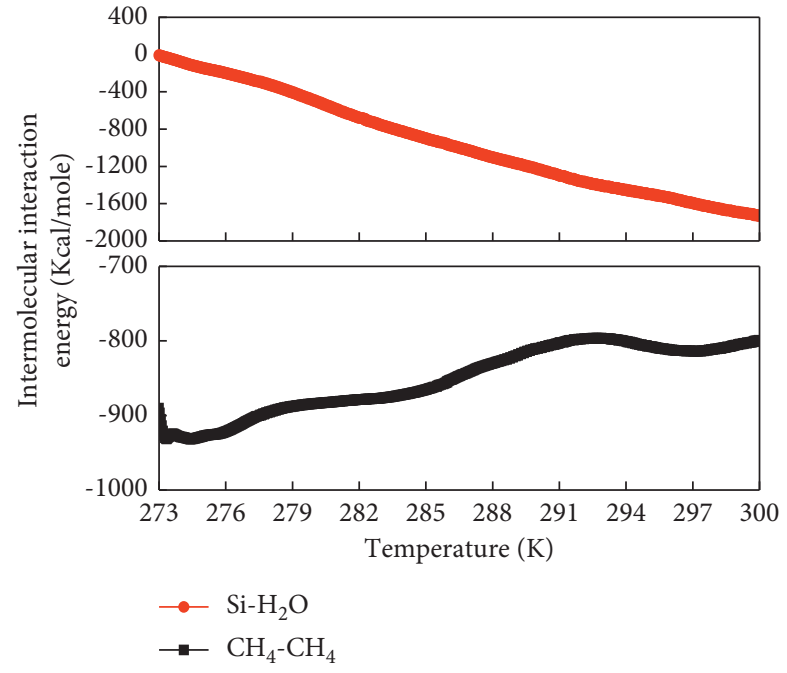

(b)

FIGURE 5: Energy evolution of MHBS during the heating process. (a) Variation curve of system energy. (b) Variation curve of intermolecular interaction energy.

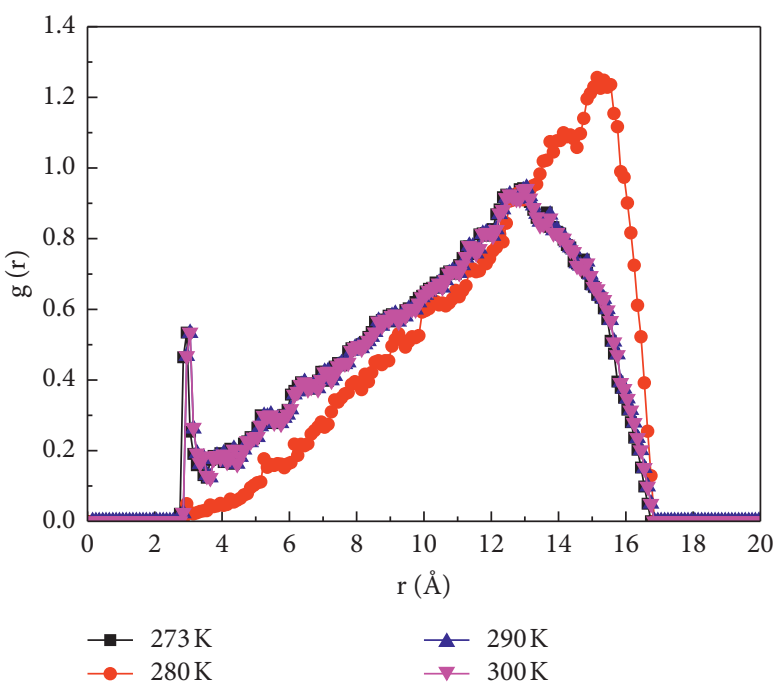

(a)

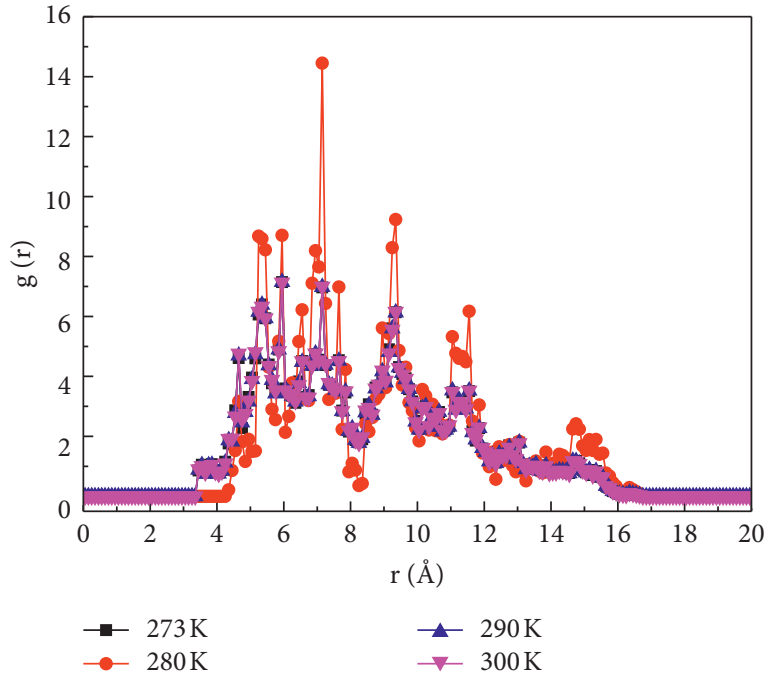

(b)

Figure 6: Radial distribution function of different atoms. (a) RDF of Si-O $\left(\mathrm{H}_{2} \mathrm{O}\right)$. (b) RDF of C-C.

during the compression process even though small deformations came out in the final compression stage. The interaction of quartz layers with water and methane molecules mainly existed at the interface of quartz layers and methane hydrate.

According to the microstructure evolution during the compression process, the migration law of methane molecules can be summarized as follows: during the compression process, the methane hydrate inside the MHBS undergoes the soil pressure from both sides, and then, the cage structures of methane hydrate are damaged, which forms the passage for the migration of methane molecules. The methane molecules on both sides and in the middle escape through the passage at the same time. However, the void spaces between the soil particles become small because of the compression. As a result, the methane molecules located in the middle part of MHBS cannot escape in time and float inside MHBS and then become air in the soil. While the fact that the damage of cage structures at the same time and the decrease in soil porosity due to compression occur simultaneously, during the compression process, the mining efficiency could be reduced.

Figure 8 shows the stress evolution of MHBS in the $y$ direction during the compression process. The stress kept decreasing and then came to a stable state. The stress decreasing rate was high at the initial stage since the dissociated methane molecules could not escape in a short time and thus caused excess pore water pressure and excess pore gas 


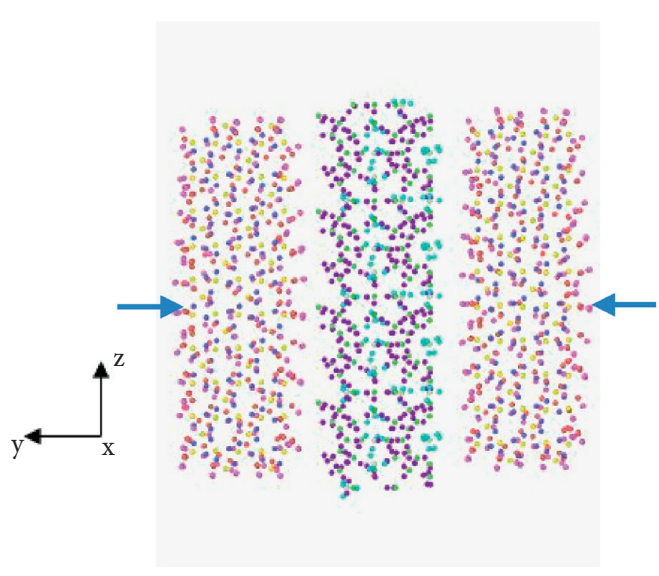

(a)

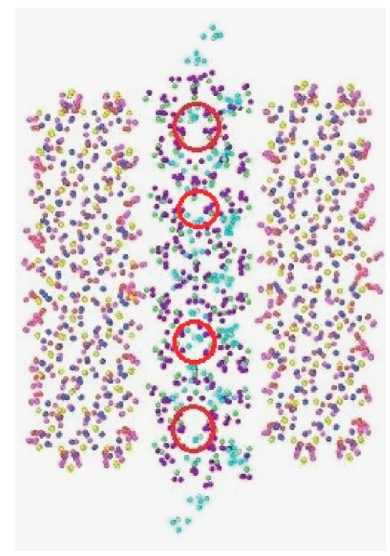

(b)

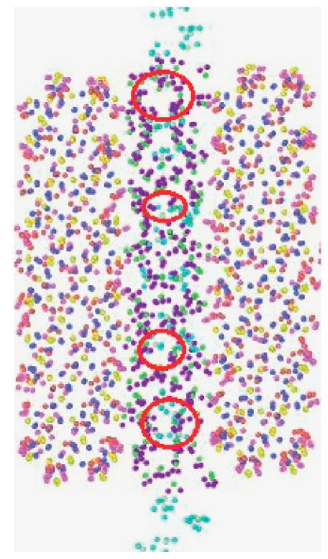

(c)

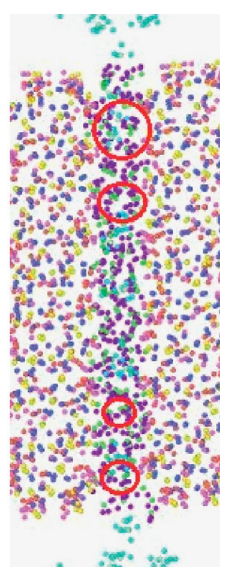

(d)

FIGURE 7: Microstructure changes of MHBS during the compression process. (a) Initial stage. (b) Methane hydrate dissociation. (c) Methane molecules escape. (d) Compression finished.

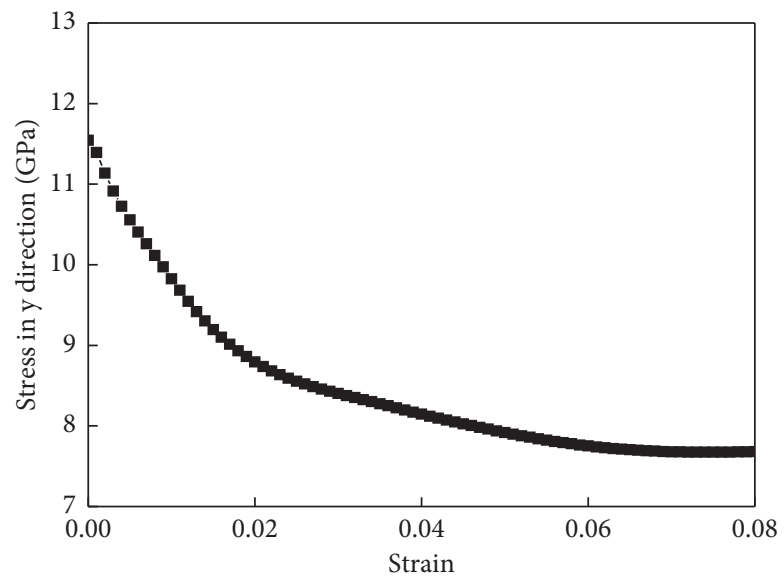

FIgURE 8: Strain-stress curve in the $y$-axis during the compression process.

pressure in the system. As the compression process went on, the methane molecules escaped through the passage continuously. The escaping methane molecules decreased the excess pore pressure in the system, which reduced the decreasing rate of microstrength of MHBS until the strength reached a stable state. Currently, the corresponding axial strain is 0.08 .

Figure 9(a) shows the energy evolution during the compression process. The energy of the total system gradually decreased during the compression process. According to the "one point" view, the dissipated energy could decrease the effective stress and the mechanical properties of MHBS.

Compared with the heating process, the energy decreased during the compression process was small. It was because methane hydrate underwent soil pressure from both sides and the void spaces in soil decreased at the same time. Under these conditions, the decomposition rate of methane hydrate decreased, and thus, the energy dissipation decreased. However, during the heating process, the methane hydrate dissociated from both sides to the middle. The molecule bonds were very sensitive to temperature variation. Thus, the methane hydrate was damaged and decomposed at a high rate. In addition, the molecule bonds of quartz layers were also affected, which was another reason for decreasing the energy dissipation rate.

Figure 9(b) shows the intermolecular interaction energy variation between silicon atoms and water molecules, and methane molecules and methane molecules during the compression process. The interaction energy between silica atoms and water molecules increased with the compression process which reduced the soil particle friction which was similar to the heating process. The interaction energy between methane molecules and methane molecules decreased slightly at a steadily decreasing rate. It was due to the migration and escape of methane molecules through the passage of MHBS. The interaction came to a relatively stable state. However, the continuous escape of the methane molecules made the interaction decrease.

From the compression process, it could be inferred that the decomposed water molecules and methane molecules could not migrate outside in time and thus led to increasing pore water pressure and pore gas pressure in the MHBS. At the same time, the escape of the methane molecules decreased the saturation of methane hydrate. Meanwhile, the 


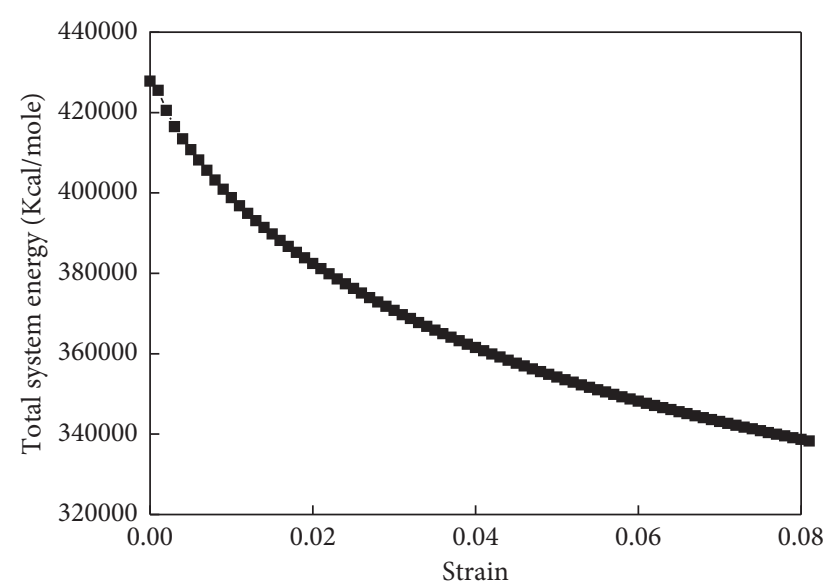

(a)

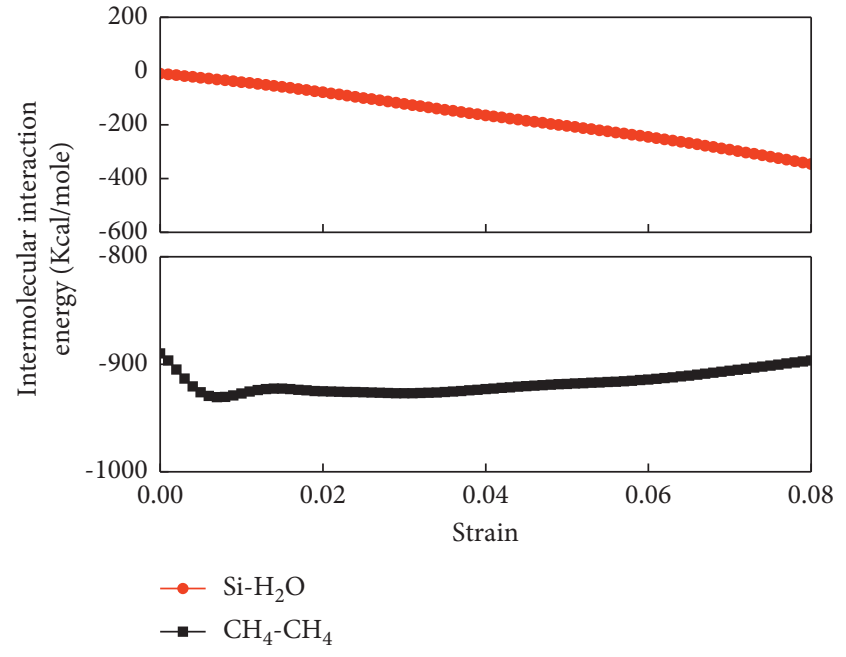

(b)

FIgURE 9: Energy evolution of MHBS during the compression process. (a) Variation curve of system energy. (b) Variation curve of intermolecular interaction energy.

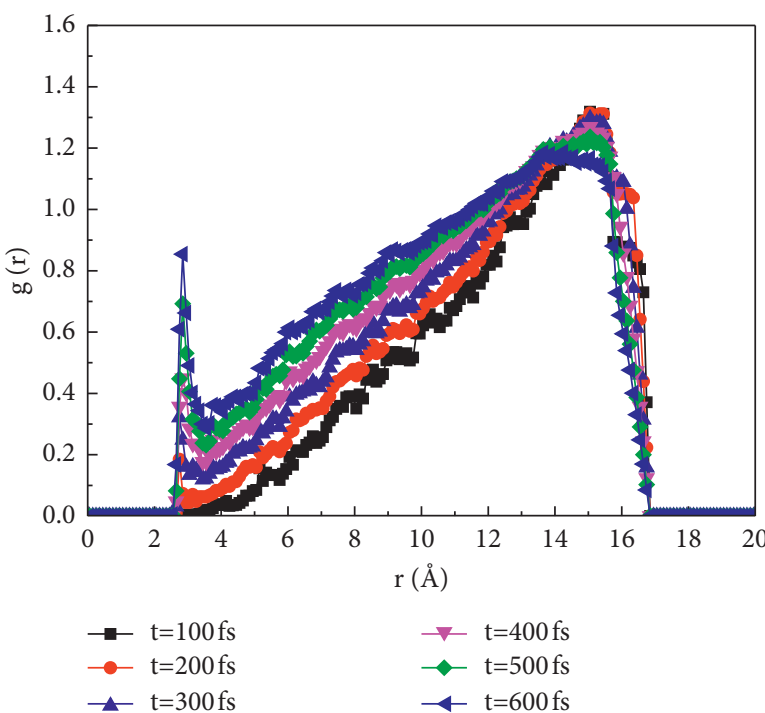

(a)

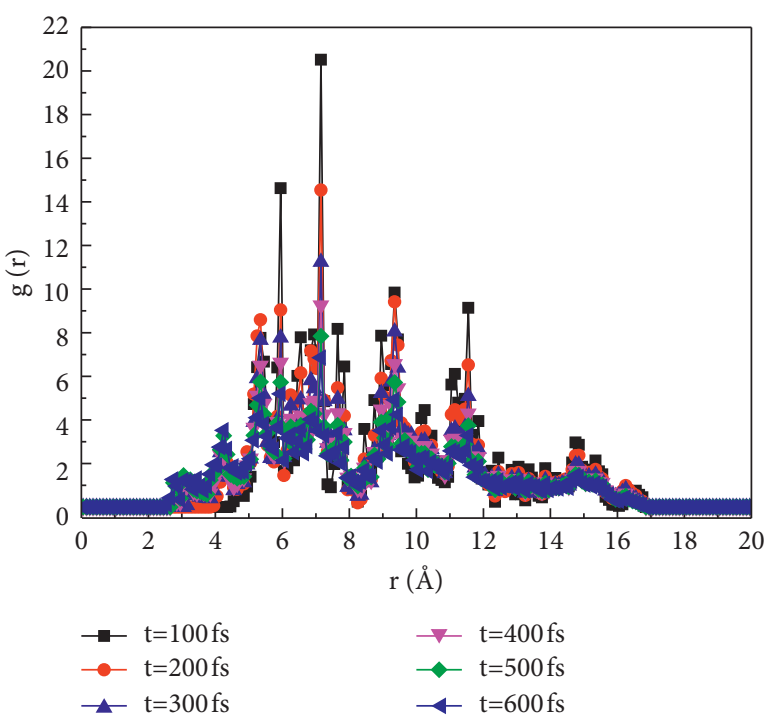

(b)

Figure 10: Radial distribution function of different atoms. (a) RDF of $\mathrm{Si}-\mathrm{O}\left(\mathrm{H}_{2} \mathrm{O}\right)$. (b) RDF of C-C.

effective stress decreased due to the accumulation of the dissipated energy in the soil. In addition, the free water molecules increased due to the methane hydrate decomposition. All these three aspects have led to the deterioration of MHBS strength.

Figure 10(a) shows RDF of silica atoms in quartz and oxygen atoms in the water molecules in the water molecule of hydrate. It has a similar trend to Figure 6 . According to $\mathrm{RDF}$, the water molecules surrounding the quartz layers kept increasing. and two peak values were observed. The peak values were higher than the values in Figure 6, which was caused by the compression of quartz layers on methane hydrate and the distance between two quartz layers becoming smaller continuously. Even so, the interactions for the compression and heating processes were different. During the heating process, the movement rate of free water molecules generated from methane hydrate became faster and interacted with the quartz layer. It was the water molecules that impacted the quartz layers. In the compression process, the free water molecules were generated from the methane hydrate damaged by the compression of quartz layers. In this process, the quartz molecules impacted the water molecules.

Figure 10(b) shows the carbon atom distribution, which reflected the distribution of methane molecules. There were multiple peaks, which indicated that methane molecules 
were tightly packed. At $10 \mathrm{w}$ steps, MHBS was just at the beginning of the methane hydrate dissociation and RDF had the highest value. As the compression process continued, the methane molecules began to escape, and the peak value became decreasing. It was consistent with the fact that the methane molecules migrate and escape only through the passage in the $Z$-direction.

\section{Conclusions}

In this paper, the microscopic mechanism of the effects of the methane hydrate phase transition on the mechanical properties of MHBS under heating and compression was studied by the MD method. The evolutions of MHBS's microstructure, system energy, intermolecular interaction energy, and RDF during the dissociation process were investigated. The detailed conclusions are summarized as follows:

(1) With the rising temperature, the methane hydrate dissociation starts from both sides to the middle and the void spaces in soil have nearly no change. Under compression, the methane hydrate on both sides and the middle dissociates at the same time, and the void spaces become smaller.

(2) During the heating and compression processes, the methane hydrates inside MHBS decompose. The methane molecules and water molecules generated migrate along the passage formed by the damaged cage structures and MHBS's mechanical properties deteriorate consequently.

(3) The microscopic mechanism of the effects of methane hydrate dissociation on the mechanical properties of the MHBS is mainly caused by three aspects. (1) The dissociation of methane hydrate incurs the decrease of methane hydrate saturation. The free water and methane molecules generated cannot migrate in time and thus lead to the increase of excess pore water pressure and the excess pore gas pressure. (2) The dissipated energy causes the decrease of the effective stress between the soil particles. (3) Due to the methane hydrate decomposition, the free water molecules increase, which reduces the friction of soil particles.

\section{Data Availability}

Some or all data, models, or codes that support the findings of this study are available from the corresponding author upon reasonable request.

\section{Conflicts of Interest}

The authors have no conflicts of interest to declare.

\section{Acknowledgments}

This work was supported by the National Natural Science Foundation of China (No. 51208510).

\section{References}

[1] H. Huneke and T. Mulder, Deep-sea Sediments, Elsevier, 2010.

[2] X. Dai, T. Xu, and J. Chen, "Physical and Mechanical Properties of Deep Oceanic Sediments Cored from the Bottom of Challenger Deep Mariana Trench," Geofluids, vol. 2021, Article ID 9109132, 11 pages, 2021.

[3] E. D. Sloan, "Fundamental principles and applications of natural gas hydrates," Nature, vol. 426, no. 6964, pp. 353-359, 2003.

[4] J. Kondori, Modeling Investigation of Gas Hydrate Decomposition: Thermodynamic Approach and Molecular Dynamic Simulations, Doctoral (PhD)thesis, Memorial University of Newfoundland, Canada, CA, 2019.

[5] K. Nakashima, Y. Nakata, M. Hyodo, N. Yoshimoto, S. Hiraoka, and S. Kajiyama, "Compressive characteristics of methane hydrate-bearing sands under isotropic consolidation," Soils and Foundations, vol. 61, no. 2, pp. 506-519, 2021.

[6] T. H. Kwon, G. C. Cho, and J. C. Santamarina, "Gas hydrate dissociation in sediments: pressure-temperature evolution," Geochemistry, Geophysics, Geosystems, vol. 9, no. 3, 2008.

[7] P. Talling, "Large submarine landslides on continental slopes: geohazards, methane release, and climate change," Oceanography, vol. 27, no. 2, pp. 32-45, 2014.

[8] S. Kataoka, S. Yamashita, T. Suzuki, and T. Kawaguchi, "Soil properties of the shallow type methane hydrate-bearing sediments in the Lake Baikal," in Proceedings of the 17th International Conference on Soil Mechanics and Geotechnical Engineering, October 2009.

[9] J. C. Santamarina, "Hydro-bio-geomechanical properties of hydrate-bearing sediments from Nankai Trough," Marine and Petroleum Geology, vol. 66, pp. 434-450, 2015.

[10] C. Ruppel, R. Boswell, and E. Jones, "Scientific results from Gulf of Mexico gas hydrates Joint Industry Project Leg 1 drilling: introduction and overview," Marine and Petroleum Geology, vol. 25, no. 9, pp. 819-829, 2008.

[11] F. Francisca, T. Ruppel, and J. C. Santamarina, "Geophysical and geotechnical properties of near-seafloor sediments in the northern Gulf of Mexico gas hydrate province," Earth and Planetary Science Letters, vol. 237, no. 3-4, pp. 924-939, 2005.

[12] S. Kajiyama, Y. Wu, M. Hyodo, Y. Nakata, K. Nakashima, and N. Yoshimoto, "Experimental investigation on the mechanical properties of methane hydrate-bearing sand formed with rounded particles," Journal of Natural Gas Science and Engineering, vol. 45, pp. 96-107, 2017.

[13] Y. Wu, N. Li, M. Hyodo, M. Gu, J. Cui, and B. F. Spencer, "Modeling the mechanical response of gas hydrate reservoirs in triaxial stress space," International Journal of Hydrogen Energy, vol. 44, no. 48, Article ID 26698, 2019.

[14] R. Holtzman, D. B. Silin, and T. W. Patzek, "Mechanical properties of granular materials: a variational approach to grain-scale simulations," International Journal for Numerical and Analytical Methods in Geomechanics, vol. 33, no. 3, pp. 391-404, 2009.

[15] Y. Wu, M. Hyodo, and J. Cui, "On the critical state characteristics of methane hydrate-bearing sediments," Marine and Petroleum Geology, vol. 116, Article ID 104342, 2020.

[16] G. Moridis and J. Rutqvist, "Numerical studies of geomechanical stability of hydrate bearing sediments," in Proceedings of the 2007 Offshore Technology Conference, Houston, X, USA, May 2007.

[17] W. Xu and L. N. Germanovich, "Excess pore pressure resulting from methane hydrate dissociation in marine 
sediments: a theoretical approach," Journal of Geophysical Research: Solid Earth, vol. 111, no. B1, 2006.

[18] Y. Jiang and B. Gong, "Discrete-element numerical modelling method for studying mechanical response of methane-hydrate-bearing specimens," Marine Georesources \& Geotechnology, vol. 38, no. 9, pp. 1082-1096, 2020.

[19] C. Li, "Investigation on the multiparameter of hydratebearing sands using nano-focus X-ray computed tomography," Journal of Geophysical Research: Solid Earth, vol. 124, no. 3, pp. 2286-2296, 2019.

[20] T. J. Kneafsey, L. Tomutsa, G. J. Moridis et al., "Methane hydrate formation and dissociation in a partially saturated core-scale sand sample," Journal of Petroleum Science and Engineering, vol. 56, no. 1-3, pp. 108-126, 2007.

[21] K. T. Pickering and R. N. Hiscott, Deep marine Systems: Processes, Deposits, Environments, Tectonics and Sedimentation, John Wiley \& Sons, Hoboken, NJ, USA, 2015.

[22] H. Ji, D. Chen, C. Zhao, and G. Wu, "Molecular dynamics simulation of methane hydrate formation and dissociation in the clay pores with fatty acids," Journal of Physical Chemistry C, vol. 122, no. 2, pp. 1318-1325, 2018.

[23] K. Li, B. Chen, Y. Song, and M. Yang, "Molecular dynamics simulation of the effects of different thermodynamic parameters on methane hydrate dissociation: an analysis of temperature, pressure and gas concentrations," Fluid Phase Equilibria, vol. 516, Article ID 112606, 2020.

[24] Q. Wang, Q. Tang, and S. Tian, "Molecular dynamics simulation of sI methane hydrate under compression and tension," Open Chemistry, vol. 18, no. 1, pp. 69-76, 2020.

[25] Y. T. Tung and L. Chen, "The growth of structure I methane hydrate from molecular dynamics simulations," The Journal of Physical Chemistry B, vol. 114, no. 33, Article ID 10804, 2010.

[26] Y. Luan, B. Liu, P. Hao, K. Zhan, and J. Liu, "Oil displacement by supercritical $\mathrm{CO} 2$ in a water cut dead-end pore: molecular dynamics simulation," Journal of Petroleum Science and Engineering, vol. 188, Article ID 106899, 2020.

[27] J. McCaughan, S. Iglauer, and F. Bresme, "Molecular dynamics simulation of water/CO2-quartz interfacial properties: application to subsurface gas injection," Energy Procedia, vol. 37, pp. 5387-5402, 2013.

[28] A. A. Skelton, P. Fenter, J. D. Kubicki, D. J. Wesolowski, and P. T. Cummings, "Simulations of the quartz(1011)/water interface: a comparison of classical force fields, ab initio molecular dynamics, and X-ray reflectivity experiments," Journal of Physical Chemistry C, vol. 115, no. 5, pp. 2076-2088, 2011.

[29] H. J. C. Berendsen, J. R. Grigera, and T. P. Straatsma, "The missing term in effective pair potentials," Journal of Physical Chemistry, vol. 91, no. 24, pp. 6269-6271, 1987.

[30] R. C. Selley, L. R. M. Cocks, and I. R. Plimer, Encyclopedia of Geology, Elsevier Academic, Amsterdam, 2005. 